\title{
Synthesis, Optical and Surface Properties of the New Gemini Quaternary Ammonium Surfactants Containing DSD Acid- triazine Structure
}

\author{
Maosheng Wan ${ }^{\mathrm{a}}$, Jinzhao Chen ${ }^{\mathrm{a}}$, Xinchun Shen ${ }^{\mathrm{a}}$, and \\ Chengbo $\mathrm{Cao}^{\mathrm{a}, *}$ \\ ${ }^{a}$ School of Chemistry and Chemical Engineering, \\ Shandong University \\ Jinan, Shandong, 250100, China
}

\author{
Jinglan Guo ${ }^{\mathrm{b}}$ \\ ${ }^{\mathrm{b}}$ Biology Institute of Shandong Academy of Sciences \\ Jinan, Shandong, 250014, China
}

\begin{abstract}
Four novel gemini quaternary ammonium surfactants containing DSD acid-triazine structure were synthesized using a facile three-step synthetic route, from 4,4'-diamino-2,2'-disulfonic-stilbene (DSD acid), cyanuric chloride and dimethyl dodecylamine. The structures and optical properties of compounds (6a-d) were characterized by Fourier-Transform infrared (FTIR) spectroscopy, UV-visible absorption spectra and fluorescence emission spectrum. The surface tension and the Critical Micelle Concentration (CMC) were evaluated. The result shows that the four compounds at lower concentrations can greatly reduce the surface tension of aqueous solutions. The spectroscopic properties of these quaternary ammonium salts are assessed for their effectiveness and potential as fluorescent brightening agents.
\end{abstract}

Keywords-Quaternary ammonium, Surfactants, DSD acid, Critical micelle concentration

\section{Introduction}

Quaternary ammonium compounds (QACs) as surfactants [1-2] and antibacterial activity [3-6] are widely used in dyestuff, textile, medicine Industry and scientific research. Particularly, those owning two quaternary ammonium nitrogen atoms called gemini surfactants, seem to exhibit more effective antimicrobial activities. Because of the greater propensity to form micelles and the further efficient decrease of surface tension compared with the single-chain surfactant corresponding conventional counterparts, gemini surfactants were attracting growing attention. As well, DSD acid-triazine structure compounds (called fluorescent brightening agents) absorb light in the near ultraviolet region of the spectrum and emit the light of violet-blue fluorescence in the visible region $(430-440 \mathrm{~nm})$ [7-8], which were widely used for whitening textile, paper and biological staining [9].

In this paper, we have reported the full details of syntheses of gemini surfactants with long chain quaternary ammonium group on triazine moiety. The newly synthesized compounds were prepared from DSD acid, cyanuric chloride and dimethyl dodecylamine in 3 steps with $80-85 \%$ overall yield. The structures and optical properties of obtained compounds were characterized. In addition, the surface tension and the Critical Micelle Concentration (CMC) of the four compounds (6a-d) were evaluated.

\section{Experimental}

\section{A. Synthesis of compound 3}

The synthetic course is shown in Scheme 1. Characterization data as shown in Table. 1. Cyanuric chloride (1) (18.4 g, $0.1 \mathrm{~mol}$ ) was added to the mixture solution of dimethyl dodecylamine (2) $(21.3 \mathrm{~g}, 0.1 \mathrm{~mol})$ in water $(1000 \mathrm{~mL})$ at $0^{\circ} \mathrm{C}$. After the addition was completed, the reaction mixture was adjusted with $10 \%$ sodium hydroxide to maintain $\mathrm{pH} 5$ and stirred for $2.5 \mathrm{~h}$ at $0-5^{\circ} \mathrm{C}$. Then the precipitate was filtered and dried to obtain compound 3 in $95 \%$ yield.<smiles>CCN(C)CC</smiles><smiles>C[N+](C)(C)c1nc(Cl)nc(Cl)n1</smiles>

4

(ii)

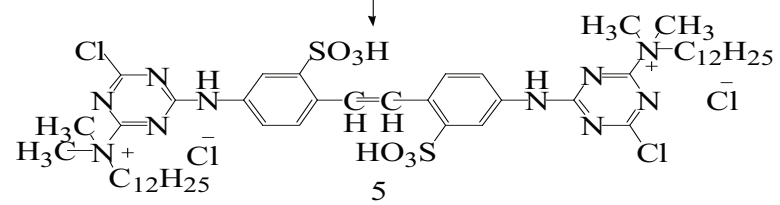
(iii)

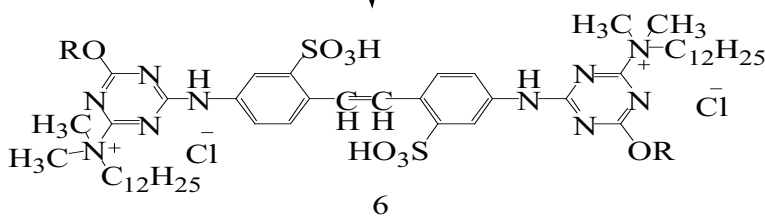

Scheme 1. Synthesis of compounds 
Reagents and conditions: (i) $\mathrm{H}_{2} \mathrm{O}, 0-5^{\circ} \mathrm{C}, 2.5 \mathrm{~h}$; (ii) $\mathrm{NaOH}, \mathrm{H}_{2} \mathrm{O}, 40-45^{\circ} \mathrm{C}, 4 \mathrm{~h}$, then $\mathrm{HCl}$ aq; (iii) a. $\mathrm{NaOH}, \mathrm{H}_{2} \mathrm{O}, 100^{\circ} \mathrm{C}, 5 \mathrm{~h}$ b. $\mathrm{RX}, 100^{\circ} \mathrm{C}, 5 \mathrm{~h}$, then $\mathrm{rt}, \mathrm{HCl}$ aq.

\section{B. Synthesis of compound 5}

Compound (3) (15.9 g, $40 \mathrm{mmol})$ was added a solution of DSD acid (4) (7.4 g, $20 \mathrm{mmol})$ and $\mathrm{NaOH}(1.6 \mathrm{~g}, 40 \mathrm{mmol})$ in water $(400 \mathrm{~mL})$ at the room tempreture. After the addition was completed, the reaction mixture was warmed to $40-45{ }^{\circ} \mathrm{C}$ and stirred for $4 \mathrm{~h}$. The reaction mixture was transferred to a beaker and acidified to $\mathrm{pH} 5$ with $4 \mathrm{M} \mathrm{HCl}$ solution. The precipitate was filtered and dried to obtain compound 5 in $96 \%$ yield.

\section{Synthesis of compounds 6a-d}

$\mathrm{NaOH}(0.21 \mathrm{~g}, 5.2 \mathrm{mmol})$ was added a solution of compound $5(2.84 \mathrm{~g}, 2.6 \mathrm{mmol})$ in water $(150 \mathrm{~mL})$. The reaction mixture was stired and warmed to $100{ }^{\circ} \mathrm{C}$ for $5 \mathrm{~h}$. Halohydrocarbon $(5.2$ mmol) was added to the reaction mixture at $100{ }^{\circ} \mathrm{C}$ for $5 \mathrm{~h}$. The reaction mixture was transferred to a beaker and acidified to pH 6 with $4 \mathrm{M} \mathrm{HCl}$ solution. The precipitate was filtered and dried to obtain compounds (6a-d) in $80-85 \%$ overall yield. TABLE 1. CHARACTERIZATION DATA OF THE COMPOUNDS

\begin{tabular}{|c|c|c|l|}
\hline Entry & $\mathbf{R}$ & Yield (\%) & $\mathbf{I R}\left(\mathbf{K B r}, \mathbf{c m}^{-1}\right)$ \\
\hline 6a & $\mathrm{CH}_{3} \mathrm{CH}_{2}$ & 81 & $3498,3212,1702$ \\
\hline 6b & $\mathrm{PhCH}_{2}$ & 85 & $3424,3212,1702$ \\
\hline 6c & $\mathrm{CH}_{2}=\mathrm{CHCH}_{2}$ & 84 & $3484,3209,1701$ \\
\hline 6d & $\bigwedge-\mathrm{CH}_{2}$ & 80 & $3426,3212,1702$ \\
\hline
\end{tabular}

\section{Results and discussion}

\section{UV/visible absorption and Fluorescence measurements}

Steady-state fluorescence spectra were recorded on a Perkin Elmer, L50 spectrofluorimeter instrument. All experiments were carried out using freshly prepared solutions containing $2 \times 10-2 \mathrm{~g} / \mathrm{L}$ of the compounds (6a-d) in deionized water and adjusting solution to $\mathrm{pH} 9$ with $\mathrm{NaOH}$ aq, using a $10 \mathrm{~mm}$ quartz cuvette. As shown in Fig. 1, compounds (6a-d) have characteristic absorptions in the range of $300 \sim 400 \mathrm{~nm}$. The emitting fluorescence of compounds (6a-d) locate in the range of $400 \sim 500 \mathrm{~nm}$ as shown in Fig. 2 .

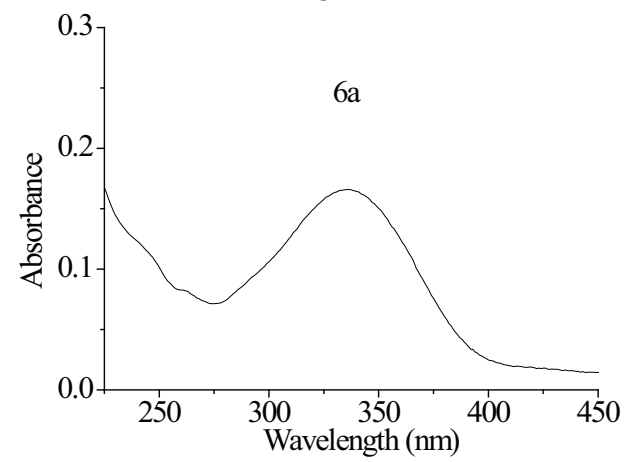

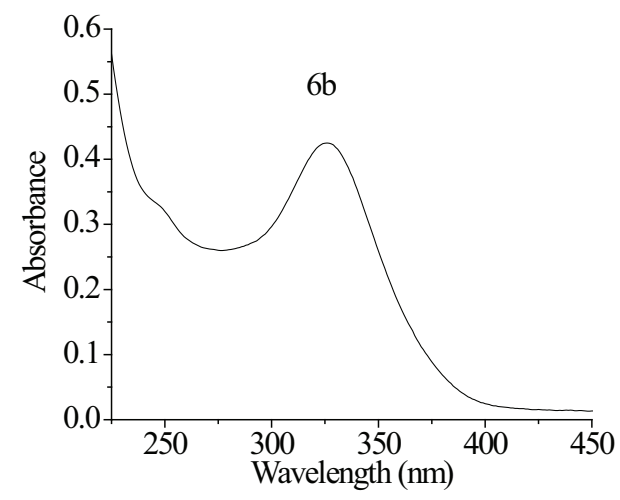
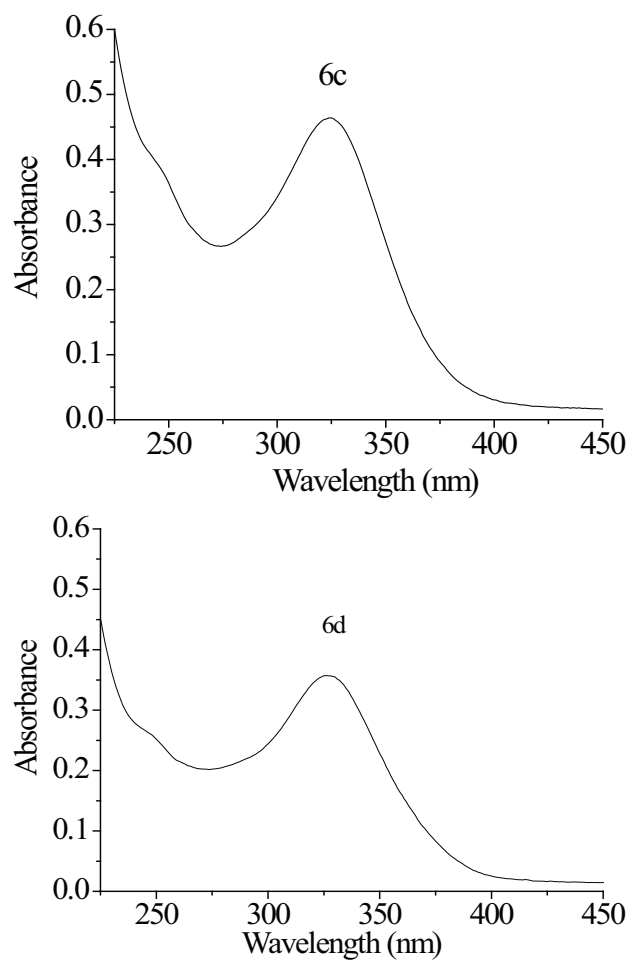

Fig. 1. UV/visible absorption spectra of compounds (6a-d)

From the Fig.1, we can know that there are one isomer in the ultraviolet region, each has its maximum absorption. The four compounds in aqueous solution have trans-isomer maximum absorption wavelength at $326 \sim 344 \mathrm{~nm}$ and a cisisomer has not been found.

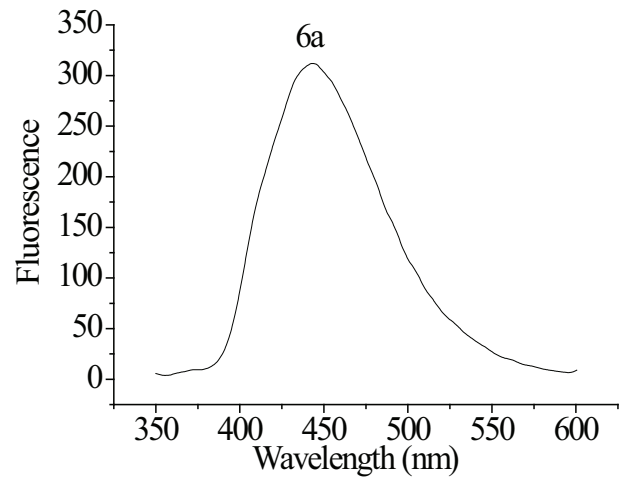

Identify applicable sponsor/s here. (sponsors) 

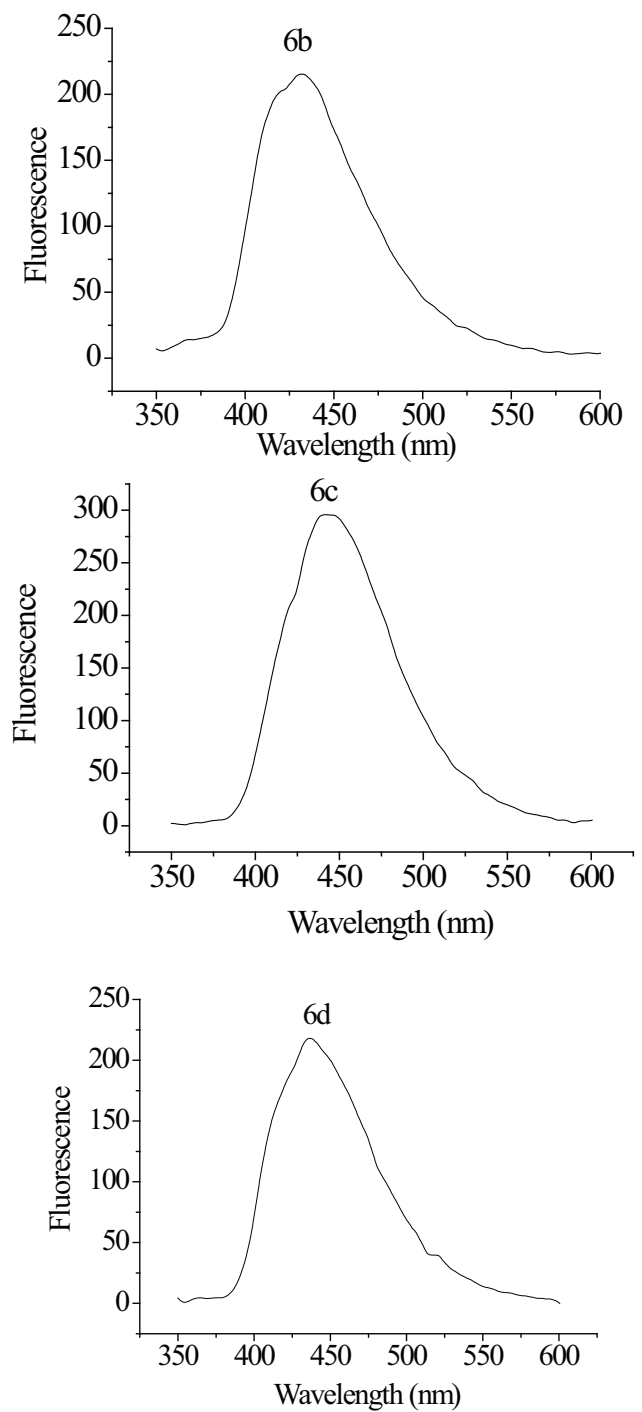

Fig.2. Fluorescence emission spectra of compounds 6a-d

From the Fig.2, The four compounds in aqueous solution emit fluorescence in the $370 \sim 540 \mathrm{~nm}$, the most intense between the $433 \sim 450 \mathrm{~nm}$. Because of the common structure, the products have the similar shape of fluorescence emission spectra curve. the products have the similar shape of fluorescence emission spectra curve.

$\mathrm{UV} /$ visible absorption and fluorescence emission maxima of the compounds as shown in Table. 2. From the Fig.1 and Fig.2, the compounds (6a-d) can be used as fluorescent brightening agents.

TABLE 2. ABSORPTION AND FLUORESCENCE DATA OF THE COMPOUNDS

\begin{tabular}{|c|c|c|c|c|}
\hline Compound & $\mathbf{6 a}$ & $\mathbf{6 b}$ & $\mathbf{6 c}$ & $\mathbf{6 d}$ \\
\hline$\lambda_{\mathrm{abs}}(\mathrm{nm})$ & 383 & 344 & 326 & 327 \\
\hline$\lambda_{\mathrm{fl}}(\mathrm{nm})$ & 443 & 443 & 445 & 437 \\
\hline
\end{tabular}

\section{E. Surface tension measurements}

The surface tensions $(\gamma)$ of aqueous solutions of the surfactants (6a-d) were measured by the Du Nouy ring method at $303 \mathrm{~K}$. The surface tension was measured three times for each sample with a 40 minute interval to ensure that the data was equilibrium between each reading. The critical micelle concentration (CMC) values were determined using a series of aqueous solutions of the surfactants at various concentrations, and estimated from the break point of each surface tension versus concentration on curves. The values of surface tension decrease continuously critical micelle concentration (CMC) values were determined using a series of aqueous solutions of the surfactants at various concentrations, and estimated from the break point of each surface tension versus concentration on curves. The values of surface tension decrease continuously and then become almost constant along a wide concentration range as shown in Fig. 3.
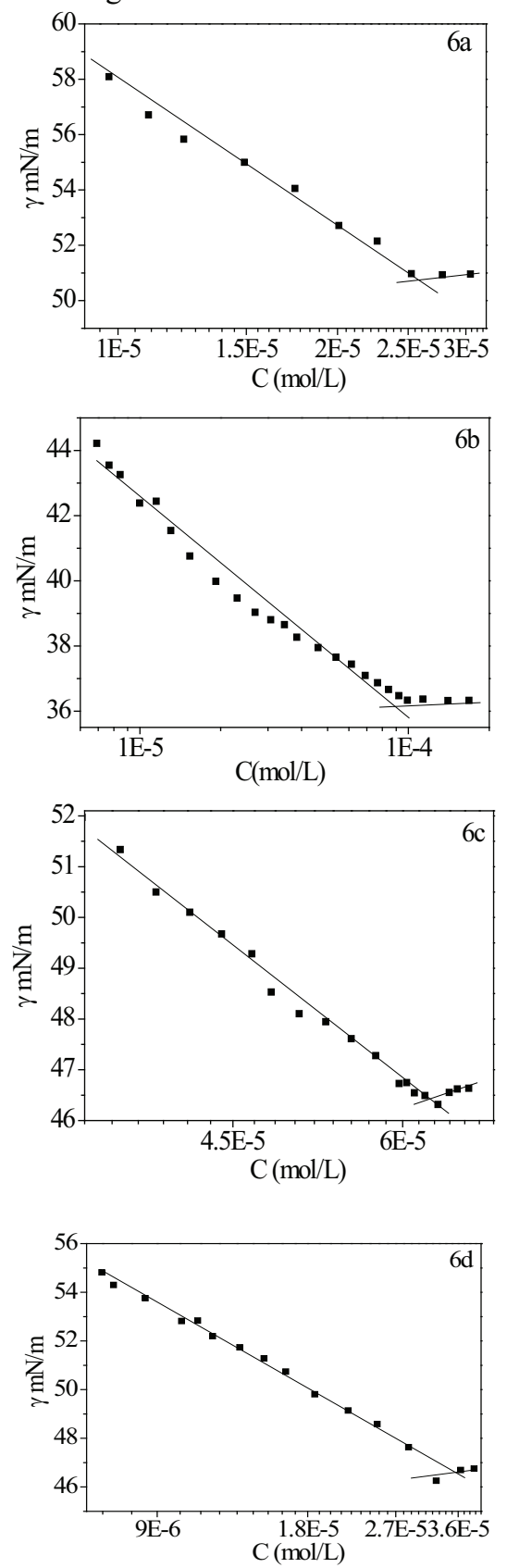

Fig. 3. Variation in the surface tension with the concentration for 6a-d 
The ability of the surfactants to lower surface tension at the CMC ( $\gamma \mathrm{cmc})$ was calculated, The $\gamma \mathrm{cmc}$ and CMC values are shown in Table 3. The instrument was calibrated against double distilled water at the time of measurement.

TABLE 3. THE TCMC AND CMC VALUES OF COMPOUNDS (6A-D)

\begin{tabular}{|c|c|c|c|c|}
\hline Compound & $\mathbf{6 a}$ & $\mathbf{6 b}$ & $\mathbf{6 c}$ & $\mathbf{6 d}$ \\
\hline$\gamma \mathrm{cmc}\left(\mathrm{mN} \mathrm{m}^{-1}\right)$ & 50.93 & 36.47 & 46.30 & 46.50 \\
\hline $\mathrm{CMC}\left(\mathrm{mol} \mathrm{L}^{-3}\right)$ & $2.6 \times 10^{-5}$ & $9.0 \times 10^{-5}$ & $6.3 \times 10^{-5}$ & $3.63 \times 10^{-5}$ \\
\hline
\end{tabular}

Generally surfactant refers to a class of compounds which can greatly decrease the surface tension in lower concentration aqueous solution compared with other solutes. With experimental detection, these four compounds called surfactant of quaternary ammonium salt showed remarkable ability to reduce the surface tension in the low concentration aqueous solution. The surface tension and surface activity of surfactant were considered and proved to be related to many factors such as the kind of compensation ion, electrostatic interaction, electrostatic shielding effect, the countra-ion combination, temperature and other element. Among the four compounds we synthetized, that their countra-ions are all chloridion, however with the different substituent groups attaching the parent structure, lead to the difference of $\gamma \mathrm{cmc}$ and $\mathrm{CMC}$ measured value between the compounds. The measured value of $\gamma \mathrm{cmc}$ of the compound whose substituent group is benzene ring is the minimum as well as that the one whose substituent group is double bond or epoxy compound is the maximum. The existence of benzene ring who is non-polar group gives rise to the sparse arrangement in the water surface of the surfactant, and changes the ability of interfacial adsorption which is attributed to the strong hydrophobic role between benzene rings. Therefore, this has the lower surface activity compared with other higher ones. But among the four compounds, this has the highest surface activity as the strongest ability of reducing the surface tension of the aqueous solution. Both the two types of compounds with epoxide group and double bond have the similar measured value of $\gamma \mathrm{cmc}$ because of the closed electrostatic shielding effect, the similar countra-ion combination, the minor difference of the ion volume, the nearly equal ability of interfacial adsorption and the similar degree of difficulty to form micelle of the two, which is consistent with the test results. Consequently, this type of compound is a growing in hydrophobic material researching.

\section{Conclusions}

Through a simple three-step condensation reaction, we obtained the four new gemini quaternary ammonium surfactants containing DSD acid-triazine structure with high yields. The obtained compounds were characterized by UVvisible absorption spectra, fluorescence emission spectra and IR spectroscopy. The surface tension and the Critical Micelle Concentration (CMC) were investigated. The result showed that the compounds (6a-d) have better optical performance as fluorescent brightening agents and can greatly reduce the surface tension of aqueous solutions at lower concentration as new gemini surfactants.

\section{REFERENCES}

[1] C.H. Jou: Colloids and Surfaces B: Biointerfaces. Vol. 88 (2011), p. 448-454.

[2] H.Q. Li, C.C. Yu, R. Chen, J. Li and J.X. Li: Colloids and Surfaces A:

Physicochem. Eng. Aspects. Vol. 395 (2012), p. 116-124.

[3] L.M. Campos, K.L. Killops, R. Sakai, J.M.J. Paulusse, D. Damiron, E.

Drockenmuller, B.W. Messmore and C.J. Hawker: Macromolecules. Vol. 41 (2008), p. 7063-7070.

[4] A. Colomer, A. Pinazo, M.A. Manresa, M.P. Vinardell, M. Mitjans, M.R.

Infante and L. Pérez: J. Med. Chem. Vol. 54 (2011), p. 989-1002.

[5] M. El Kateb, E. Taffin de Givenchy, A. Baklouti and F. Guittard: J.

Colloid Interface Sci. Vol. 357 (2011), p. 129-135.

[6] L. Caillier, E.T. de Givenchy, R. Levy, Y. Vandenberghe, S. Geribaldi

and F. Guittard: J. Colloid Interface Sci. Vol. 332 (2009), p. 201-207.

[7] H. Zollinger: Color chemistry. 3rd ed (2003), p. 365.

[8] J.K. Lee, S.I. Um, Y. Kang and D.J. Baek: Dyes Pigments. Vol. 64 (2005), p. 25-30.

[9] B.J. Harrington: Lab Medicine. Vol. 40 (2009), p. 219223. 